\title{
Mass number dependence in $\Lambda$-hypernuclei by means of the Hypervirial Theorems
}

\author{
Th.E.Lioliosin \\ Department of Theoretical Physics,University of Thessaloniki,Thessaloniki 54006, Greece
}

\begin{abstract}
The Hypervirial Theorems are applied to a wide class of single particle nuclear potentials, in order to study the mass number dependence of various quantities in $\Lambda$-hypernuclei. A very efficient model is assumed for the radius parameter of the potentials and the limitations of the whole method are discussed.
\end{abstract}

PACS: 21.80.+a, 13.75.Ev, 21.10.Dr

\section{Introduction}

Over the past years a variety of methods has been used for the determination of the $\Lambda$-hyperon binding energies in hypernuclei, ranging from that of nuclear emulsions to the strangeness exchange reaction [1, 2, 3] up to the more recent associated production

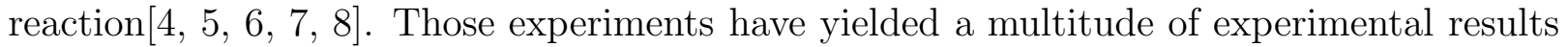
which allow for a better description of the mass number dependence of the $\Lambda$-binding energies. Moreover, this wealth of data provides a solid foundation of an analytical approach of $\Lambda$-hypernuclei. Various single particle potentials have been used in the past in order to approximate the self-cosistent potenial in a $\Lambda$-hypenucleus 19, 10, 11, 12, 13, 14], while their parameters have been studied systematically by means of the associated production reaction $\left(\pi^{+}, K^{-}\right)$. The results indicate that the depth of the potential can be safely regarded as mass number independent (except for very light nuclei) with a value close to $30 \mathrm{MeV}$ 114, 15, 16, 17, 18]. However, it turns out that mass number dependence has to be taken seriously into account for a nuclear potential to reproduce the experimental data satisfactorily. With data available on the spacing of single particle levels in a given hypernuclei, one can also determine the functional dependence of the radius parameter on the mass number, thus constructing a very efficient potential model.

In addition, in a recent work, the Hypervirial Theorems (HVT) were applied to a wide class of single patricle nuclear potentials yielding various valuable approximate quantities [19, 20, 21, 22]. Hence, by incorporating a mass number dependent radius parameter in that work a thorough study of $\Lambda$-hypernuclei can be accomplished.

\section{Application of the hypervirial theorems to single particle nuclear poten- tials.}

A wide class of potentials used in Nuclear Physics is :

$$
V(r)=-D f\left(\frac{r}{R}\right) \quad 0 \leq r<\infty
$$

where $D$ is the potential depth, $R=r_{0} A^{\frac{1}{3}}$ the potential radius, $r_{0}$ the radius parameter and $A$ the mass number of the nucleus. The "potential form factor" f, $(f(0)=1)$, is an

†email:theoliol@ccf.auth.gr 
even analytic function of $x=\frac{r}{R}$. Namely,

$$
f(x)=\sum_{k=0}^{\infty} d_{k} x^{2 k}
$$

where $d_{k}$ are the numbers:

$$
d_{k}=\left.\frac{1}{(2 k) !} \frac{d^{2 k}}{d x^{2 k}} f(x)\right|_{x=0} \quad k=0,1,2, \ldots \quad d_{1}<0
$$

The corresponding Schroedinger eigenvalue problem is:

$$
\begin{gathered}
{\left[\frac{d^{2}}{d x^{2}}-\frac{l(l+1)}{x^{2}}+s^{-2} f(x)+\tilde{E}_{n l}\right] u_{n l}(x)=0} \\
u_{n l}(0)=0 \quad u_{n l}(\infty)=0
\end{gathered}
$$

where, $x=\frac{r}{R}, s=\left(\frac{\hbar^{2}}{2 \mu D R^{2}}\right)^{\frac{1}{2}}$ and $\widetilde{E}_{n l}=s^{-2} \frac{E_{n l}}{D}$

An application of the hypervirial theorems to(4) yielded fairly accurate series for the corresponding energy eigenvalues $E_{n l}$, the potential $\langle V\rangle_{n l}$ and the kinetic energy $\langle T\rangle_{n l}$ and the mean-square radius (MSR) $<r^{2}>_{n l}$. By observing[22] that those quantities are actually exclusive function of the parameter $s$ one can write those series in the form:

$$
\begin{gathered}
E_{n l}=D \sum_{k=0}^{\infty} e_{n l}^{(k)} s^{k} \quad, \quad V>_{n l}=D \sum_{k=0}^{\infty} v_{n l}^{(k)} s^{k}, \quad<T>_{n l}=D \sum_{k=0}^{\infty t} t_{n l}^{(k)} s^{k} \\
<r^{2}>_{n l}=R^{2} \sum_{k=0}^{\infty} r_{n l}^{(k)} s^{k}
\end{gathered}
$$

where

$$
t_{n l}^{(k)}=\left(\frac{1+k}{2}\right) e_{n l}^{(k)} \quad, \quad v_{n l}^{(k)}=\left(\frac{1-k}{2}\right) e_{n l}^{(k)}
$$

The first significant coefficients $e_{n l}^{(k)}$ are :

$$
\begin{gathered}
e_{n l}^{(0)}=-1 \quad, \quad e_{n l}^{(1)}=2 a_{n l}\left(-d_{1}\right)^{\frac{1}{2}} \quad, \quad e_{n l}^{(2)}=\frac{d_{2}}{8 d_{1}}\left[12 a_{n l}^{2}-4 l(l+1)+3\right] \\
e_{n l}^{(3)}=-\frac{a_{n l}\left(-d_{1}\right)^{\frac{1}{2}}}{32 d_{1}^{3}}\left\{4 a_{n l}^{2}\left(20 d_{1} d_{3}-17 d_{2}^{2}\right)+4 d_{1} d_{3}[25-12 l(l+1)]+d_{2}^{2}[36 l(l+1)-67]\right\}
\end{gathered}
$$

Likewise, the corresponding first significant coefficients for the MSR are:

$$
r_{n l}^{(0)}=0 \quad, \quad r_{n l}^{(1)}=\frac{a_{n l}}{\left(-d_{1}\right)^{\frac{1}{2}}} \quad, \quad r_{n l}^{(2)}=\frac{d_{2}}{8 d_{1}^{2}}\left[12 a_{n l}^{2}-4 l(l+1)+3\right]
$$


$r_{n l}^{(3)}=-\frac{a_{n l}\left(-d_{1}\right)^{\frac{1}{2}}}{64 d_{1}^{4}}\left\{20 a_{n l}^{2}\left(12 d_{1} d_{3}-17 d_{2}^{2}\right)+12 d_{1} d_{3}[25-12 l(l+1)]+5 d_{2}^{2}[36 l(l+1)-67]\right\}$

III. Derivation of a general formula for mass number dependent quantities.

Potentials of the form (便) have been used extensively in Hypernuclear Physics with very satisfactory results. Nevertheless, mass number dependence has to be assumed for the radius parameter $r_{0}$ as well, for a reliable reproduction of the experimental results, that is:

$$
R=r_{0}(A) A^{\frac{1}{3}}
$$

where $A$ the mass number of the core nucleus, assuming the rigid core model. Such an assumption effectively implies that the volume each nucleon occupies $\left(\frac{4}{3} \pi r_{0}^{3}\right)$ as well as the mean interparticle distance $\left(2 r_{0}\right)$ are functions of $A$, too. According to some very thorough studies in the field of hypernuclei the experimental results can be fitted much better by a single particle potential if the radius parameter $r_{0}$ is assumed to have the following form [9, 11]:

$$
r_{0}=\left(r_{1}+r_{2} A^{-\frac{2}{3}}\right)
$$

On the basis of this assumption a member of the potential class(1) was employed to study the functional dependence of binding energies, kinetic and potential energies in $\Lambda$-hypernuclei[1]. That study can now be generalised, by means of the HVT, for the entire class(11), including the study of the MSR.

The parameter $s$ appearing in the previous section can now be written :

$$
s=s_{\Lambda} \frac{\sqrt{1+g A^{-1}}}{A^{\frac{1}{3}}\left(1+b A^{-\frac{2}{3}}\right)},
$$

where the new parameters introduced are:

$$
s_{\Lambda}=\sqrt{\frac{\hbar^{2}}{2 m_{\Lambda} D r_{0}^{2}}}, \quad b=\frac{r_{2}}{r_{1}}, \quad g=\frac{m_{\Lambda}}{m_{N}}
$$

$m_{N}, m_{\Lambda}$ are the masses of the nucleon and of the $\Lambda$-particle respectively.

By expanding $s$ in terms of $A^{-\frac{1}{3}}$ and dropping terms of order higher than $A^{-1}$ the following approximation is arrived at:

$$
s \simeq s_{\Lambda}\left(A^{-\frac{1}{3}}-b A^{-1}\right)
$$

Consequently the expectation value of a quantity $Q$, using the HVT formalism, takes the approximate form:

$$
<Q>_{n l}=\sum_{k=0}^{\infty} q_{n l}^{(k)} s^{k} \simeq \sum_{k=0}^{\infty} q_{n l}^{(k)} s_{\Lambda}^{k}\left(A^{-\frac{1}{3}}-b A^{-1}\right)^{k}
$$

Neglecting higher terms, the above approximation is finally written as follows: 


$$
<Q>_{n l} \simeq q_{n l}^{(0)}+2 s_{0} q_{n l}^{(1)} A^{-\frac{1}{3}}+4 s_{0}^{2} q_{n l}^{(3)} A^{-\frac{2}{3}}+\left(8 s_{0}^{3} q_{n l}^{(3)}-2 b s_{0} q_{n l}^{(1)}\right) A^{-1}
$$

where $s_{0}=\frac{s_{\Lambda}}{2}$

By replacing $q_{n l}^{(k)}$ in the above expression with the corresponding coefficients $e_{n l}^{(k)}, v_{n l}^{(k)}, t_{n l}^{(k)}$, $r_{n l}^{(k)}$, the mass number dependence of $E_{n l},<V>_{n l},<T>_{n l}$, and $\left\langle r^{2}>_{n l}\right.$ assumes a very enlightening quantitative form for each state.

As $g$ vanishes in the present approximation, for $b=0$ formula (16) corresponds to the conventional model introduced by the nuclear radius $R=r_{0} A^{\frac{1}{3}}$.

At this venture some comments regarding the MSR are deemed necessary as the importance of the $A$ dependence of the energy quantities is obvious, anyway. Although there are no experimental data for the MSR of a $\Lambda$-particle,its usefullness becomes apparent in the calculation of the oscillator level spacing according to the approximate formula:

$$
\hbar \omega_{\Lambda}=\frac{3}{2} \frac{\hbar^{2}}{\mu}<r^{2}>_{00}^{-1}
$$

A thorough study of the validity of the above formula was undertaken [13] using the reduced Poeschl-Teller potential which behaves very much alike the Gaussian one, with some extra analytic features.

Moreover, the oscillator spacing can be used in the calculation of the relative probability of the recoiless $\Lambda$-production using the Debye-Waller factor [14]:

$$
P\left(n_{i}, n_{i}\right) \simeq \exp \left[\frac{-\left(2 n_{i}+1\right) \hbar^{2} q^{2}}{2 m_{\Lambda} \hbar \omega_{\Lambda}}\right]
$$

where $n_{i}$ is the quantum number of the harmonic oscillator state and $\hbar q$ the $\Lambda$-recoil momentum.

For the potential model used in [13, 14] the calculation of $\hbar \omega_{\Lambda}$ as a function of $A$ was easy and performed in a straightforward way. In general, however, one has to resort to a special treatment of potentials (11), such as that of the HVT which yields Eq.(6) .

Hence, an assessment of Eq.(6) with respect to existing experimental data can only be accomplished indirectly, through the oscillator spacing and only for the ground state. Alternatively, a numerical integration of the Schroedinger equation(4⿴囗十) can also provide a measure of its accuarcy 19, 20, 21]. The orbital radius of a $\Lambda$-particle, taken as the root mean square radius RMSR $=\sqrt{\langle r\rangle_{n l}^{2}}$, can now be written as a fraction of the potential radius:

$$
\sqrt{<r>_{n l}^{2}}=F(A) R \quad, \quad F(A)=\sqrt{\sum_{k=0}^{\infty} r_{n l}^{(k)} s^{k}}<1
$$

\section{Implementation of of the derived formulae and their limitations}

Two typical members of (四) are the Gaussian potential :

$$
V(r)=-D e^{-\frac{r^{2}}{R^{2}}}
$$


for which

$$
d_{k}=\frac{(-1)^{k}}{k !}
$$

and the (reduced) Poeschl-Teller potential

$$
V(r)=-D \cosh ^{-2}(r / R) .
$$

As for the Gaussian potential the previous formalism is readily applied to it to obtain the mass number dependence of the binding energies :

$$
\begin{gathered}
E_{1 s}=-D\left[1-6 s_{0} A^{-\frac{1}{3}}+\frac{15}{2} s_{0}^{2} A^{-\frac{2}{3}}+\left(\frac{25}{8} s_{0}^{3}+6 b s_{0}\right) A^{-1}\right] \\
E_{1 p}=-D\left[1-10 s_{0} A^{-\frac{1}{3}}+\frac{35}{2} s_{0}^{2} A^{-\frac{2}{3}}+\left(\frac{105}{8} s_{0}^{3}+10 b s_{0}\right) A^{-1}\right] \\
E_{1 d}=-D\left[1-14 s_{0} A^{-\frac{1}{3}}+\frac{63}{2} s_{0}^{2} A^{-\frac{2}{3}}+\left(\frac{273}{8} s_{0}^{3}+14 b s_{0}\right) A^{-1}\right] \\
E_{2 s}=-D\left[1-14 s_{0} A^{-\frac{1}{3}}+\frac{75}{2} s_{0}^{2} A^{-\frac{2}{3}}+\left(\frac{315}{8} s_{0}^{3}+14 b s_{0}\right) A^{-1}\right]
\end{gathered}
$$

Similarly one obtains the potential and kinetic energy mass number dependence:

$$
\begin{gathered}
V_{1 s}=-D\left[1-3 s_{0} A^{-\frac{1}{3}}-\left(\frac{25}{16} s_{0}^{3}-3 b s_{0}\right) A^{-1}\right] \\
V_{1 p}=-D\left[1-5 s_{0} A^{-\frac{1}{3}}-\left(\frac{105}{16} s_{0}^{3}-5 b s_{0}\right) A^{-1}\right] \\
T_{1 s}=D\left[3 s_{0} A^{-\frac{1}{3}}-\frac{15}{2} s_{0} A^{-\frac{2}{3}}-\left(\frac{75}{16} s_{0}^{3}+3 b s_{0}\right) A^{-1}\right] \\
T_{1 p}=D\left[5 s_{0} A^{-\frac{1}{3}}-\frac{35}{2} s_{0} A^{-\frac{2}{3}}-\left(\frac{375}{16} s_{0}^{3}+5 b s_{0}\right) A^{-1}\right]
\end{gathered}
$$

The above relations are identical to the ones obtained in a thorough study [1] of the applicability of (20) to $\Lambda$-hypernuclei assuming the mass number dependence for the radius parameter given by (11). In that work the validity of (11) was firmly established by the substantial agreement between the numerical values of the analytic expressions and the corresponding experimental data.

The present approach can also provide the MSR for various states:

$$
\begin{aligned}
& <r^{2}>_{1 s}=R^{2}\left[3 s_{0} A^{-\frac{1}{3}}+\frac{405}{32} s_{0}^{2} A^{-\frac{2}{3}}+\frac{3\left(135 s_{0}^{3}-16 b s_{0}\right)}{16} A^{-1}\right] \\
& <r^{2}>_{1 p}=R^{2}\left[5 s_{0} A^{-\frac{1}{3}}+\frac{1365}{32} s_{0}^{2} A^{-\frac{2}{3}}+\frac{5\left(273 s_{0}^{3}-16 b s_{0}\right)}{16} A^{-1}\right]
\end{aligned}
$$


Similar epressions can be produced for potential (22), by means of (16).

In Fig.1, Fig.2 and Fig. 3 , the binding energies of a $\Lambda$-hyperon and the corresponding potential and kinetic energies are plotted with respect to $A^{-\frac{2}{3}}$. Available experimental data [8] are also shown in Fig.1. The fitting parameters for the Gaussian potential obtained are[1] : $D=32.45 \mathrm{MeV}, r_{1}=1.438, r_{2}=-1.118$

In Fig.4, Eqs. (10) , (31), (32) (and the corresponding $<r^{2}>_{1 d}$ ) are used in order to plot the potential radius $R$ and the orbital radius of a $\Lambda$-hyperon, for various eigenstates, against

$A^{\frac{1}{3}}$. That figure is in accordance with the argument that "the distinguishable $\Lambda$-particle provides one of the best examples of single particle shell structure in nuclear physics" 9].

The method of the HVT introduces limitations to the present formalism from the very beginning. A solid criterion for the practicality of the method is the magnitude of the parameter $s$. Apparently the truncated series (16) is accurate enough only if $s$ is sufficiently small which is akin to the potential being deep and wide. In addition, previous studies have shown that the method works better for the deeply bound states of relatively heavy nuclei[19, 20, 21]. Therefore in Fig.4 the reliable region is that lying lower than the potential radius as the more the discontinuous curves approach the solid line from the right, the more loosely bound the particle becomes.

An equally important limitation is due to the potentials (1) being two-parameter ones and therefore unable to take into account some surface effects a three-parameter potential such as the Woods-Saxon one would. That inherent inadequacy renders such potentials unsuitable for the description of very heavy nuclei. On the other hand, for vey light systems the depth parameter should have also been assumed mass number dependent.

Consequently, for Eqs. (23) to (32), a safe validity range of hypernuclei is :20<A<120, whereas, for the loosely bound states, their accuracy could be slightly improved by including a higher term (e.g. of order $A^{-\frac{4}{3}}$ ).

Finally, it should be underlined that nothing prevents the present formalism from being applied to nucleon-nucleus interactions, possibly assuming a different model for the radius parameter according to experimental data.

\section{ACKNOWLEDGEMENTS}

The author would like to thank C.Daskalogiannis, S.Massen and G.Lalazissis for useful comments and discussion.

\section{References}

[1] B.Povh, Prog.Part.Nucl.Phys.5,1 (1978)

[2] W.Brückner et al., Pys.Lett. 55B, 107 (1975); 79B, 157 (1978)

[3] R.Bertini et al., Phys.Lett. 83B, 306 (1979)

[4] C.Milner et al., Phys.Rev. Lett. 54, 1237 (1985) 
[5] R.E.Chrien, Proc.of XIth Int.Conf.on Particles and Nuclei, Kyoto 1987 (Nucl.Phys.A 478, 705c (1988))

[6] P.H.Pile, Proc.of Int.Symp.on Hypernuclear and Low Kaon Physics, Padua 2988 (Nuovo Cimento A 102, 413 (1989))

[7] R.E.Chrien, ibid. Nuovo Cimento A.102, 823 (1989)

[8] P.H.Pile et al., Phys. Rev.Lett.66, 2585 (1991)

[9] D.J.Millener, C.B.Dover, and A.Gal, Phys.Rev.C 38, 2700(1988)

[10] G. A. Lalazissis,Phys. Rev. C 48,198(1993)

[11] G.A.Lalazissis ,Phys. Rev. C 49,(1994)1412, and private communication

[12] G.A.Lalazissis , J.Phys.G.Nucl.Part.Phys. 19 (1993)

[13] G.A.Lalazissis, M.E.Grypeos, S.E.Massen, Phys.Rev. C 37, 2098 (1987)

[14] M.E.Grypeos et al, Z.Phys.A,Atom.Nucl. 332, 391(1989)

[15] R.H.Dalitz in Nuclear Physics, edited by C.DeWitt and V.Gillet (Gordon and Breach, New York, 1975), Vol.8, p.1

[16] R.E.Bertini et al ., Nucl.Phys. A360, 315 (1981)

[17] A.Bouyssy and J.Hüfner, Phys.Lett.64B,276 (1976); A.Bouyssy, ibid 84B,41 (1979)

[18] A.Gal, in Advances in Nuclear Physics, edited by M.Baranger and E.Vogt (Plenum, New York, 1975) Vol8.p1

[19] Th.E.Liolios M.E.Grypeos , J.Phys.A,30(1997)L325-L330

[20] Th.E.Liolios and M.E.Grypeos International Journal of Theoretical Physics 36 (1997) 2051

[21] Th.E.Liolios, Computer Physics Communications,105(1997)254

[22] M.E.Grypeos and Th.E.Liolios submitted for publication 